\title{
Prognostic Risk Analyses for Postcardiotomy Extracorporeal Membrane Oxygenation in Children: A Review of Early and Intermediate Outcomes
}

\author{
Miki Asano ${ }^{1}$ (D $\cdot$ Hidekazu Matsumae $^{2} \cdot$ Kazutaka Suzuki $^{3} \cdot$ Yousuke Nakai $^{2} \cdot$ Takuya Nakayama $^{4} \cdot$ Norikazu Nomura $^{2}$. \\ Akira Mishima ${ }^{2}$
}

Received: 22 April 2018 / Accepted: 12 August 2018 / Published online: 21 August 2018

(c) Springer Science+Business Media, LLC, part of Springer Nature 2018

\begin{abstract}
We evaluated the morbidity and mortality of children requiring postcardiotomy extracorporeal membrane oxygenation (ECMO) to determine independent factors affecting early and intermediate outcomes. Between January 2002 and December 2015, 79 instances of ECMO after cardiac surgery in 73 children were retrospectively reviewed. Follow-up was completed in December 2016. Predictive risk analyses were employed concerning weaning of ECMO, hospital discharge, and mortality after discharge. Age and weight were $14.9 \pm 25.6$ months and $7.0 \pm 5.3 \mathrm{~kg}$, respectively. Median support time was $8.3 \pm 4.4$ days. Sixty-seven (85\%) were successfully weaned off ECMO and 48 (61\%) survived to hospital discharge. Multi-variate logistic regression analysis identified the first day to obtain negative fluid balance after initiation of support (adjusted odds ratio $=0.42$ ), high serum lactate levels $(0.97)$, and high total bilirubin $(0.84)$ during support as significant independent factors associated with successful separation from ECMO. The first day of negative fluid balance (0.65) after successful decannulation was an independent risk factor for survival to hospital discharge. After hospital discharge, actuarial 1-year, 5-year, and 10-year survival rates were $94 \%, 78 \%$, and $78 \%$, respectively. Low weight increased the risk of death after hospital discharge by a multi-variate Cox hazard model. High serum lactate, high serum bilirubin, and unable to obtain early negative fluid balance during support impacted mortality of decannulation. Obtaining a late negative fluid balance in post-ECMO were independent risk factors for death after successful weaning. Low weight affected intermediate outcomes.
\end{abstract}

Keywords Extracorporeal membrane oxygenation $\cdot$ Fluid negative balance $\cdot$ Low body weight $\cdot$ Pediatric cardiac surgery $\cdot$ Serum bilirubin $\cdot$ Serum lactate

\section{Introduction}

Extracorporeal membrane oxygenation (ECMO) is the most common form of mechanical support for children with intractable cardiopulmonary failure [1, 2]. Despite new

Miki Asano

asano@hm.tokoha-u.ac.jp

1 Faculty of Health Promotional Sciences, Tokoha University, 1230 Miyakota, Kita-ku, Hamamatsu 431-2102, Japan

2 Department of Cardiovascular Surgery, Nagoya City University, Nagoya, Japan

3 Department of Pediatrics, Nagoya City University, Nagoya, Japan

4 Division of Cardiovascular Surgery, Nagoya Kyouritsu Hospital, Nagoya, Japan innovations in pediatric ventricular assist devices (VADs) and artificial heart technologies, ECMO remains the primary modality of support for neonatal and pediatric patients. In 2016, the Extracorporeal Life Support Organization reported that 14,849 pediatric patients including 6475 neonatal patients had been supported with cardiac ECMO, which was almost twice that reported in 2009 [3, 4]. Various applications of ECMO, including cardiac support after surgery for congenital heart disease, have been reported, but mortality remains high among patients who need ECMO after surgical intervention for cardiac disease. Survival to hospital discharge of neonatal and pediatric patients has remained relatively static near $40 \%$ and $50 \%$, respectively [3-5].

Various outcomes and risk factors for successful weaning from support and hospital survival have been reported [6-11]. Although reversibility of heart dysfunction is the key determinant for ECMO success, many variables, including 
age, body weight, ventricle morphology, need for dialysis, elevated lactate levels, longer support time, timing of initiation, and water balance during support, impact ECMO survival $[6-8,10-12]$. These clinical features may be helpful to predict outcomes but have not been examined in depth. Therefore, successful weaning does not always result in survival to hospital discharge. The most common reasons for death are cardiac failure and multisystem organ failure (MOF) $[6,7,11,13]$. However, the independent risk factors for hospital survival post-ECMO and whether these factors impact prognosis after discharge remain unclear.

We investigated independent risk factors that affect successful ECMO, hospital survival, and prognosis after hospital discharge. In addition, we examined when continued ECMO was likely futile and which variables might indicate this progression. Understanding the risk factors and indicators around ECMO may aid prognosis and forestall deterioration of a patient's condition to save these critically ill children.

\section{Materials and Methods}

\section{Patients and Materials}

This study was approved by the Institutional Review Board at Nagoya City University with waiving of patient consent.

Between January 2002 and December 2015, 79 pediatric patients needed ECMO after a cardiac operation at Nagoya City University Hospital. Children who needed ECMO prior to surgical intervention or more than two ECMO procedures during the same hospitalization were excluded. The last follow-up was completed in December 2016, or at the time of death.

A retrospective analysis of perioperative clinical data and outcomes was conducted from the medical records. The following characteristics were evaluated: age and body weight at surgery related to ECMO; gender; anatomical diagnosis; surgical procedures; the time of cardiopulmonary bypass (CPB) and aortic cross clamping (AoCC); indications and total support time; systemic ventricle wall motion assessed by echocardiogram at $72 \mathrm{~h}$ of ECMO initiation or separation; the levels of serum lactate, creatinine, total bilirubin, white blood cell (WBC) count, and C-reactive protein (CRP); the total volume of urinary output within $72 \mathrm{~h}$ of initiation or separation; the number of days to obtain the first day of negative fluid balance (FNFB) after initiation or separation; use of peritoneal dialysis (PD) as a renal replacement therapy; the surgical intervention and behavior of the sepsis-related organ failure assessment (SOFA) score [14] during or after support; bleeding complications; and the number ECMO circuit exchanges during support.
Bleeding complications were defined as more than 4-5 ml $/ \mathrm{kg} / \mathrm{h}$ for more than 2 consecutive hours in the first 24 postoperative hours, surgical re-exploration for bleeding, or poor ECMO operation because of blood clots in the pericardium. Echocardiograms were conducted and evaluated by a pediatric cardiologist. Patients who discontinued ECMO or died within $72 \mathrm{~h}$ after separation were evaluated by a final echocardiogram. Quantitative evaluations were invalid because of various systemic ventricle morphologies and abnormal loading conditions under mechanical support after CPB. Therefore, a simple qualitative evaluation was used and defined by ventricle wall motion that was severe (hypokinesis)-akinesis or mild-moderate (hypokinesis). Free wall and septum (if single ventricle, bi-lateral free wall) were divided equally into three areas in the logaxis view as the basal, mid, and apex portions. A grade of severe hypokinesis-akinesis of the systemic ventricle was defined as hypokinetic areas in more than 5 out of 6 whole areas. Mild-moderate was defined as a localized hypokinetic area in 2-4 of 6 whole areas of the ventricle. Sepsis, life-threatening organ dysfunction caused by a dysregulated host response to infection, was defined as an increase in the SOFA score of 2 points or more [14]. Because of deep sedation and cardiopulmonary mechanical support, the available indicators for counting the SOFA score during ECMO were only creatinine, bilirubin, and platelets. We compared the total SOFA score at ECMO initiation with that of the highest point during the first $72 \mathrm{~h}$ of support. After ECMO, we compared the total value just after weaning with that of the highest value during the first $72 \mathrm{~h}$ of decannulation.

\section{ECMO Indications}

Indications included the inability to wean from $\mathrm{CPB}$ in the operating room (OR) and postoperative hemodynamic instability in intensive care unit (ICU) with clinical findings of hypoxemia, pulmonary hypertension $(\mathrm{PH})$, and progressive decline of cardiac function despite the maximum amount of inotropic agents or cardiopulmonary arrest. Patients were divided into two groups as follows: the group of "nonrescue" represented patients unable to wean from CPB and "rescue" included patients initiated ECMO support after successful weaning from CPB in the OR or in the ICU.

\section{ECMO Techniques}

Venoarterial ECMO was used in all patients. Direct intrathoracic cannulation was performed for patients placed on ECMO in the OR after CPB and for those placed on ECMO in the ICU. The cannulation sites were the right or common atrium for venous outflow and the aorta or neo-aorta for arterial inflow. Cannulation techniques were modified to suit specific anatomic details, particularly in patients with prior 
cavopulmonary shunts. A left heart atrial vent was employed when poor decompression of the left ventricle was noted despite adequate ECMO flow. The systemic-to-pulmonary shunt was left open to maintain shunt patency during support $[5,15]$. However, in children with decreasing systemic perfusion despite increasing ECMO flow, pulmonary blood flow was limited by adjusting the shunt diameter by a surgical clip. A standard ECMO circuit consisting of a MERA Heart-Lung-Machine HAS II small type roller pump (Senkou Ika Inc. Tokyo, Japan) and a Biocube2000/4000 membrane oxygenator (Nipro Inc. Osaka, Japan) connected with a MERA ExcelineCircuit HP2 was used. The sternum was left open and the wound covered by an expanded polytetrafluoroethylene sheet, which was further covered by sterile plastic drape with settlement of draining tubes.

For patients placed on ECMO in the OR or electively in the ICU, ECMO was initiated using a blood-primed circuit. For patients requiring urgent ECMO cannulation, such as during cardiopulmonary resuscitation (CPR), a crystalloidprimed circuit was used. Heparin and/or Nafamostat Mesilate were administered to maintain activated clotting time between 180 and $220 \mathrm{~s}$, and a range between 150 and 180 was accepted for bleeding patients. Hematocrit and platelet counts were maintained at more than $40 \%$ and more than $120,000 / \mathrm{m}^{3}$, respectively, by packed red blood cell and platelet transfusions. Fresh frozen plasma and antithrombin III were optionally administered in the presence of persistent marked mediastinal bleeding. Prophylactic antibiotics were routinely administered in all children with an open chest, and dosage was adjusted as needed. Mechanical ventilation was adjusted to prevent lung atelectasis and to maintain oxygenation of blood generated by native cardiac output. Diuretics were given to promote diuresis when urine output fell below $2-3 \mathrm{ml} / \mathrm{kg} / \mathrm{h}$. PD was used for extra volume removal in patients with oliguria or anuria. Body weight was measured every $24 \mathrm{~h}$. Enteral nutrition began $48-72 \mathrm{~h}$ after beginning ECMO unless feed was poorly absorbed by gastrointestinal edema. Body temperature was maintained at $36-37^{\circ} \mathrm{C}$ using a water mattress and convection body warmer. All patients on mechanical support were given neuromuscular blocking agents and sedated with fentanyl and/or benzodiazepine. A transthoracic follow-up echocardiography was performed at most daily. Cranial ultrasound through the anterior fontanel was performed to rule out intraventricular bleeding as appropriate. ECMO circuits and/or oxygenators were exchanged if marked thrombus occurred in the circuits, especially at the inflow site, which was examined daily, or if the oxygenator malfunctioned in patients who needed continuous ECMO support.

Discontinuation of support was predicated on either sufficient cardiopulmonary stability to wean off ECMO, or irreversible organ dysfunction that precluded survival [6]. Decannulation was decided by multiple factors including cardiopulmonary function and extravascular patient fluids. Weaning trials were essential to assess the behavior of the systemic ventricle during increases in preload, and to determine whether ECMO could be removed. Separation from ECMO was accomplished by maximizing inotropic agents including epinephrine and full ventilator support. Weaning involved a gradual decrease in flow by $10 \%$ every hour to a flow of $20 \%$ of maximal support (30-40 $\mathrm{ml} / \mathrm{kg} / \mathrm{min}$ ) with maintenance of clinical parameters. The systemic-to-pulmonary shunt, if clipped, was opened completely when flow was $50 \%$ of total flow. Gaseous nitric oxide beginning at 20-40 ppm was administered to children with refractory PH. After successful separation, the cannulae were left in place for $1 \mathrm{~h}$, flushed every $10 \mathrm{~min}$, and the recirculation line was declamped. Once the patient could maintain hemodynamic parameters, decannulation was performed. All pursestring sutures were left in place and resnared until sternal closure was performed a day after decannulation.

\section{Statistical Analysis}

Continuous variables are presented as mean \pm standard deviation. One day was converted to 0.03 months. Paired and unpaired continuous variables were compared using a Wilcoxon signed-rank test and Mann-Whitney U test, respectively. Categorical variables were analyzed by Pearson's $\chi^{2}$-test or Fisher's exact test as appropriate. A McNemar test was used to compare paired categorical variables. Logistic regression analysis was used for univariate and multivariate prognostic risk analyses for the weaning of ECMO or discharge from the hospital. Uni-variate logistic regression analysis was performed for significantly different variables between survivors and non-survivors. All covariates associated with decreased survival at $p$ values of 0.2 or less by a step-wise logistic model were extracted for the optimal combination and entered into forward selection of a multivariable logistic model for independent prognostic risk analyses. A Cox proportional hazards model was used for the risk analysis of survival after hospital discharge by evaluating all covariates that impacted survival of ECMO and/or hospital discharge in the logistic regression analysis. A step-wise logistic model was used for selecting the optimal combination of covariates for multivariate Cox hazard analysis.

Patient survival rates were calculated according to the Kaplan-Meier life table method. All statistical tests were twosided, and type I error was controlled at 0.05. Analyses were performed using JMP 10.1 (SAS Institute, Cary, NC, USA).

\section{Results}

Between January 2002 and December 2015, 79 ECMO operations in 73 children who needed cardiac surgery were performed at Nagoya City University Hospital. Six 
patients experienced ECMO runs twice for different hospitalizations. The patients on ECMO support represented $3.8 \%$ of the 2063 children undergoing surgery for congenital cardiac disease with an overall hospital mortality of $1.7 \%$. A total of 67 (85\%) were successfully weaned off ECMO and 48 (72\%) survived to hospital discharge as ECMO survivors. The overall survival to discharge was $61 \%$ in the entire cohort. The actuarial 1-year, 5-year, and 10 -year survival rates for ECMO survivors were $66 \%$, $55 \%$, and $55 \%$, respectively. After hospital discharge, the mean follow-up duration was $4.9 \pm 3.7(0.5-13.0)$ years and follow-up was completed in 47 children. The actuarial 1-year, 5-year, and 10-year survival rates after discharge were $94 \%, 78 \%$, and $78 \%$, respectively.

\section{Demographics and Pre-ECMO Variables (Table 1)}

Patients ranged in age between 1 day and 15 years with a median age of $14.9 \pm 25.6$ months. The average body weight was $7.0 \pm 5.3(2.5-37.0) \mathrm{kg}$, and the number of males was 43 (54\%). Age and sex did not differ between survivors and non-survivors nor did the time of CPB and AoCC. Body weight at the time of surgery related to ECMO was markedly lower in hospital non-survivors. There was a trend toward poorer survival in patients with mean age $<10.6$ months and a mean body weight $<5.5 \mathrm{~kg}$. Single-ventricular versus biv-entricular anatomy did not differ between survivors and non-survivors of ECMO or hospital discharge. Surgical procedure and indications of support also were not different between survivors and non-survivors of ECMO or hospital discharge. However, the rate of heterotaxy was higher in ECMO non-survivors $(p=0.0054)$.

\section{Detail of Diagnoses and Survivors (Table 2)}

Twenty-six patients had single-ventricle and 53 had bi-ventricular physiology. Heterotaxy was identified in 16 patients consisting of univentricular heart in $12(75 \%)$ and bi-ventricular heart in 4 (25\%).

No statistical differences were found in actuarial survival rates between single-ventricular and bi-ventricular hearts after hospital discharge (log-rank test, $p=0.5638$ ).

\section{Details of Surgical Procedures (Table 3)}

Surgical procedures were as follows: definitive surgery including total cavopulmonary connection (TCPC) $(n=53)$, palliative surgery $(n=26)$ consisting of Norwood stage I $(n=11)$, bi-directional cavopulmonary shunt (BCPS) $(n=4)$, systemic-pulmonary artery shunt $(n=4)$, and others $(n=7)$. Other palliative procedures with one case each were atrioventricular valve plasty, Damus-Kay-Stansel with B-T shunt, mitral valve replacement, pulmonary artery plasty, pulmonary artery plasty/pulmonary artery-right ventricle conduit, and pulmonary vein obstruction releasing. There were no significant differences in definitive or palliative surgery between survivors and non-survivors of ECMO, hospital discharge, or after hospital discharge. Actuarial survival rates among surgical procedures indicated no difference after discharge (log-rank test, $p=0.6999)$.

\section{Details of ECMO Indications (Table 4)}

Indications for ECMO were the non-rescue of patients $(n=64)$ and the rescue of patients after surgery $(n=15)$ from intractable low cardiac output condition $(n=7)$, cardiac arrest $(n=4)$, or a PH crisis $(n=4)$. In case of rescue

Table 1 Demographic and pre-ECMO variables compared between survivors and non-survivor of ECMO weaning or hospital discharge

\begin{tabular}{|c|c|c|c|c|c|c|c|c|}
\hline & \multicolumn{4}{|l|}{ ECMO } & \multicolumn{4}{|c|}{ Hospital discharge } \\
\hline & Total $(n=79)$ & Survivor $(n=67)$ & $\begin{array}{l}\text { Non-survivor } \\
(n=12)\end{array}$ & $p$ value & Total $(n=67)$ & Survivor $(n=48)$ & $\begin{array}{l}\text { Non-survivor } \\
(n=19)\end{array}$ & $p$ value \\
\hline Sex (male:female) & $43: 6$ & $38: 29$ & $5: 7$ & 0.3350 & $38: 29$ & $22: 26$ & $12: 7$ & 0.0702 \\
\hline Age (month) & $14.9 \pm 25.6$ & $15.9 \pm 27.3$ & $9.3 \pm 12.4$ & 0.4359 & $15.9 \pm 27.3$ & $18.0 \pm 30.0$ & $10.6 \pm 17.2$ & 0.0631 \\
\hline Body weight (kg) & $7.0 \pm 5.3$ & $7.3 \pm 5.6$ & $5.5 \pm 3.0$ & 0.2326 & $7.3 \pm 5.6$ & $8.2 \pm 6.3$ & $5.3 \pm 3.0$ & $0.0144 *$ \\
\hline CPB time (min) & $355 \pm 132$ & $348 \pm 136$ & $393 \pm 108$ & 0.2823 & $348 \pm 136$ & $340 \pm 132$ & $365 \pm 147$ & 0.6933 \\
\hline AoCC time (min) & $132 \pm 72$ & $136 \pm 71$ & $113 \pm 82$ & 0.3532 & $136 \pm 71$ & $131 \pm 70$ & $145 \pm 73$ & 0.6512 \\
\hline $\begin{array}{l}\text { Morphological diagno- } \\
\text { sis (single:biventricle) }\end{array}$ & $25: 54$ & $20: 47$ & $5: 7$ & 0.4177 & $20: 47$ & $15: 33$ & $5: 14$ & 0.6908 \\
\hline Heterotaxy (yes:no) & $16: 63$ & $10: 57$ & $6: 6$ & $0.0054^{*}$ & $10: 57$ & $7: 41$ & $3: 16$ & 0.9006 \\
\hline $\begin{array}{l}\text { Surgical procedure } \\
\text { (definitive:palliative) }\end{array}$ & $26: 53$ & $21: 46$ & $5: 7$ & 0.4834 & $21: 46$ & $16: 32$ & $5: 14$ & 0.5763 \\
\hline $\begin{array}{l}\text { ECMO indication (non- } \\
\text { rescue:rescue) }\end{array}$ & $64: 15$ & $55: 12$ & $9: 3$ & 0.5642 & $55: 12$ & $40: 8$ & $15: 4$ & 0.6730 \\
\hline
\end{tabular}

AoCC aortic cross clamping, $C P B$ cardiopulmonary bypass, ECMO extracorporeal membrane oxygenation

${ }^{*} p$ value $<0.05$ was considered significant 
ECMO, mean CPR time was $30.4 \pm 20.4$ min. No statistical differences were found in both ECMO and hospital survival between the two types of indications, and a trend towards poor outcomes occurred for rescue ECMO.

\section{Variables During ECMO and Post-ECMO (Tables 5, 6)}

Several variables during ECMO were markedly different between ECMO survivors and non-survivors. Furthermore, some variables differed between hospital survivors and nonsurvivors. Post-ECMO variables that differed between hospital survivors and non-survivors were as follows: WBC, lactate, creatinine, urinary output, FNFB, PD deployment, and echocardiogram result.

\section{Behavior of Variables in Hospital Survivors and Non-survivors (Table 7)}

The period of hospitalizations after ECMO was $62.2 \pm 68.4$ (2-240) days and 67.3 $\pm 44.1(18-193)$ days in survivors and non-survivors, respectively $(p=0.1599)$. Increased WBC and CRP was observed in both groups. Survivors had markedly increased urinary volume and a decreased rate of PD employment and grade of severe-akinesis in wall motion. In non-survivors, there was a marked increase of creatinine.
Table 2 Anatomical diagnosis and survivor

Table 3 Surgical procedures and survivors

Table 4 ECMO indications and survivors

\begin{tabular}{llrcrc}
\hline Morphology & Diagnosis & Number & \multicolumn{2}{l}{ Number of survivor } \\
\cline { 4 - 6 } \cline { 4 - 5 } Single-ventricular & HLHS & & ECMO & Hospital discharge & After discharge \\
& Non-HLHS & 20 & $17(85 \%)$ & $13(65 \%)$ & $3(50 \%)$ \\
& Total & 26 & $21(81 \%)$ & $16(61 \%)$ & $10(50 \%)$ \\
Biventricular & L-R & 25 & $23(92 \%)$ & $20(80 \%)$ & $17(68 \%)$ \\
& Left obstructed & 4 & $3(75 \%)$ & $2(50 \%)$ & $1(25 \%)$ \\
& Cyanosis PA/PS & 10 & $7(70 \%)$ & $6(60 \%)$ & $6(60 \%)$ \\
& Cyanosis congestion & 12 & $11(92 \%)$ & $3(25 \%)$ & $2(17 \%)$ \\
& Others & 2 & $2(100 \%)$ & $1(50 \%)$ & $0(0 \%)$ \\
& Total & 53 & $46(87 \%)$ & $32(60 \%)$ & $26(49 \%)$ \\
\hline
\end{tabular}

ECMO extracorporeal membrane oxygenation, $H L H S$ hypoplastic left heart syndrome, $L-R$ left to right shunted heart, $P A / P S$ pulmonary atresia/pulmonary stenosis

\begin{tabular}{llcccc}
\hline Surgical procedure & Number & \multicolumn{2}{l}{ Number of survivors } \\
\cline { 3 - 5 } & & & ECMO & Hospital discharge & After discharge \\
\hline Definitive surgery & Total & 53 & $46(87 \%)$ & $32(60 \%)$ & $27(51 \%)$ \\
Palliative surgery & Norwood stage I & 11 & $8(73 \%)$ & $6(55 \%)$ & $3(27 \%)$ \\
& BCPS & 4 & $4(100 \%)$ & $3(75 \%)$ & $2(50 \%)$ \\
& Systemic-pulmonary shunt & 4 & $2(50 \%)$ & $2(50 \%)$ & $2(50 \%)$ \\
& Others & 7 & $7(100 \%)$ & $5(71 \%)$ & $5(71 \%)$ \\
& Total & 26 & $21(81 \%)$ & $16(61 \%)$ & $12(46 \%)$ \\
\hline
\end{tabular}

ECMO extracorporeal membrane oxygenation, $B C P S$ bidirectional cavopulmonary shunt

\begin{tabular}{llcccc}
\hline \multirow{2}{*}{ ECMO indication } & Number & \multicolumn{2}{l}{ Number of survivors } \\
\cline { 5 - 5 } & & & ECMO & Hospital discharge & After discharge \\
\hline None-rescue & Total & 64 & $55(86 \%)$ & $40(63 \%)$ & $31(48 \%)$ \\
Rescue & Low cardiac output & 7 & $7(100 \%)$ & $6(86 \%)$ & $6(86 \%)$ \\
& Cardiac arrest & 4 & $3(75 \%)$ & $1(25 \%)$ & $1(25 \%)$ \\
& PH crisis & 4 & $2(50 \%)$ & $1(25 \%)$ & $1(25 \%)$ \\
& Total & 15 & $12(80 \%)$ & $8(53 \%)$ & $8(53 \%)$ \\
\hline
\end{tabular}

ECMO extracorporeal membrane oxygenation, $\mathrm{PH}$ crisis pulmonary hypertension crisis 
Table 5 During ECMO variables comparing between survivor and non-survivor of ECMO or hospital discharge

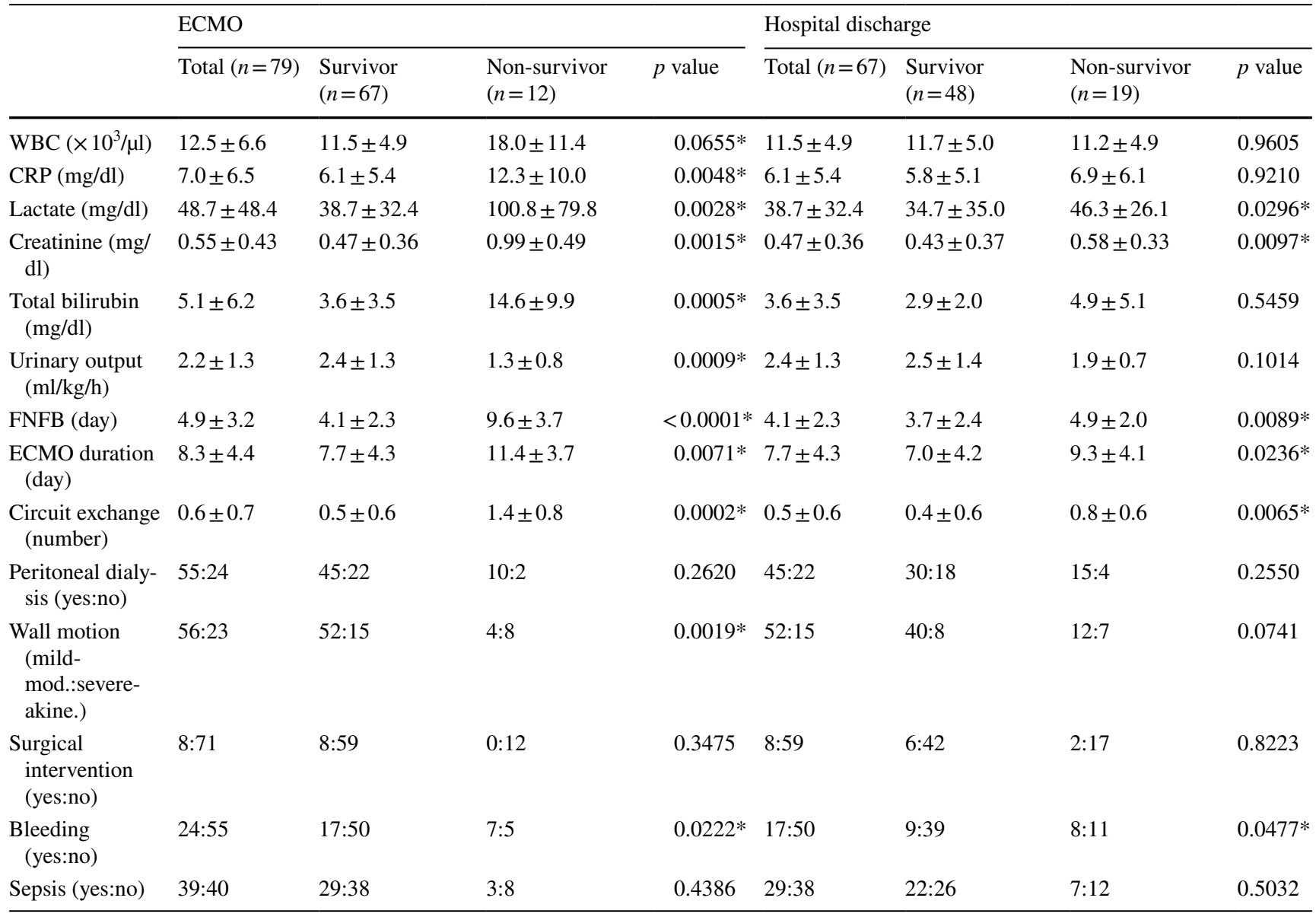

$C R P$ C-reacting protein, ECMO extracorporeal membrane oxygenation, $F N F B$ the day of first negative fluid balance, mild-mode. mild-moderate hypokinesis, severe-akine. severe hypokinesis-akinesis, $W B C$ white blood cell

* $p$ value $<0.05$ was considered significant

Table 6 Post-ECMO variables comparing between survivor and non-survivor of hospital discharge

\begin{tabular}{|c|c|c|c|c|}
\hline & \multicolumn{4}{|c|}{ Hospital discharge } \\
\hline & Total $(n=67)$ & Survivor $(n=48)$ & Non-survivor $(n=19)$ & $p$ value \\
\hline $\mathrm{WBC}\left(\times 10^{3} \mu / \mathrm{ml}\right)$ & $20.1 \pm 9.3$ & $18.4 \pm 7.4$ & $22.0 \pm 7.5$ & $0.0298^{*}$ \\
\hline CRP (mg/dl) & $7.6 \pm 5.8$ & $7.4 \pm 4.4$ & $8.3 \pm 8.6$ & 0.5455 \\
\hline Lactate (mg/dl) & $30.5 \pm 25.3$ & $23.3 \pm 15.6$ & $43.9 \pm 33.8$ & $0.0094 *$ \\
\hline Creatinine (mg/dl) & $0.78 \pm 0.72$ & $0.54 \pm 0.45$ & $1.23 \pm 0.91$ & $0.0011 *$ \\
\hline Total bilirubin (mg/dl) & $4.4 \pm 4.8$ & $3.8 \pm 3.7$ & $5.7 \pm 6.5$ & 0.4040 \\
\hline Urinary output (ml/kg/h) & $3.3 \pm 2.6$ & $4.3 \pm 2.6$ & $1.6 \pm 1.2$ & $<0.0001 *$ \\
\hline FNFB (day) & $3.9 \pm 6.4$ & $2.1 \pm 1.5$ & $8.2 \pm 10.2$ & $0.0004^{*}$ \\
\hline Peritoneal dialysis (yes:no) & $34: 33$ & $16: 32$ & $18: 1$ & $<0.0001 *$ \\
\hline Wall motion (mild-mod.:severe-akine.) & $61: 6$ & $46: 2$ & $15: 4$ & $0.0291 *$ \\
\hline Surgical intervention (yes:no) & $4: 63$ & $3: 45$ & $1: 18$ & 0.8779 \\
\hline Sepsis (yes:no) & $20: 47$ & $12: 36$ & $8: 11$ & 0.1679 \\
\hline
\end{tabular}

$C R P$ C-reacting protein, $E C M O$ extracorporeal membrane oxygenation, $F N F B$ the day of first negative fluid balance, mild-mod. mild-moderate hypokinesis, severe-akine. severe hypokinesis-akinesis, $W B C$ white blood cell

$* p$ value $<0.05$ was considered significant 
Table 7 Behavior of variables between during ECMO and post-ECMO in hospital survivor or non-survivor

\begin{tabular}{|c|c|c|c|c|c|c|}
\hline & \multicolumn{3}{|c|}{ Hospital survivor $(n=48)$} & \multicolumn{3}{|c|}{ Hospital non-survivor $(n=19)$} \\
\hline & During ECMO & Post-ECMO & $p$ value & During ECMO & Post-ECMO & $p$ value \\
\hline $\mathrm{WBC}\left(\times 10^{3} \mu / \mathrm{ml}\right)$ & $11.7 \pm 5.0$ & $18.4 \pm 7.5$ & $<0.0001^{*}$ & $11.2 \pm 4.9$ & $22.3 \pm 7.5$ & $<0.0001 *$ \\
\hline $\mathrm{CRP}(\mathrm{mg} / \mathrm{dl})$ & $5.8 \pm 5.1$ & $7.4 \pm 4.4$ & $0.0014 *$ & $6.9 \pm 6.1$ & $8.3 \pm 5.7$ & 0.2293 \\
\hline Lactate (mg/dl) & $34.7 \pm 35.0$ & $23.3 \pm 15.6$ & 0.9900 & $46.3 \pm 26.1$ & $43.9 \pm 33.8$ & 0.6738 \\
\hline Creatinine (mg/dl) & $0.4 \pm 0.4$ & $0.5 \pm 0.4$ & 0.0918 & $0.6 \pm 0.3$ & $1.2 \pm 0.9$ & $0.0002 *$ \\
\hline Urinary output (ml/kg/h) & $2.5 \pm 1.4$ & $4.3 \pm 2.6$ & $0.0001 *$ & $1.9 \pm 0.7$ & $1.6 \pm 1.2$ & 0.8710 \\
\hline FNFB (day) & $3.7 \pm 2.4$ & $2.1 \pm 1.5$ & 0.9988 & $4.9 \pm 2.0$ & $8.3 \pm 10.2$ & 0.4409 \\
\hline Peritoneal dialysis (yes:no) & $30: 18$ & $16: 32$ & $0.0010^{*}$ & $15: 4$ & $18: 1$ & 0.0833 \\
\hline Wall motion (mild-mod.:severe-akine.) & $40: 8$ & $46: 2$ & $0.0012 *$ & $12: 7$ & $15: 4$ & 0.0750 \\
\hline
\end{tabular}

CRP C-reacting protein, ECMO extracorporeal membrane oxygenation, FNFB the day of first negative fluid balance, mild-mod. mild-moderate hypokinesis, severe-akine. severe hypokinesis-akinesis, $W B C$ white blood cell

* $p$ value $<0.05$ was considered significant

\section{Outcome of ECMO or Hospital Discharge}

Successful separation from ECMO was not accomplished in 12 patients. Elective withdrawal of support occurred in 10 patients and a PH crisis in 2 immediately after weaning. Withdrawal indications included non-recovery of myocardial function $(n=6)$, multi organ failure (MOF) with sepsis $(n=3)$, and brain death by cerebral bleeding $(n=1)$.

Nineteen children were unable to survive to discharge after decannulation because of MOF $(n=9)$, LOS $(n=6)$, sepsis $(n=2)$, cardiac arrest $(n=1)$, and hypoxia encephalopathia $(n=1)$. Six MOF patients were associated with sepsis. CNS complications $(n=3)$, including intracranial hemorrhage, hypoxic insult, or brain death, not described in the table were not different between ECMO survivors and non-survivors (2/67 (3.0\%) and 1/12 (8.3\%), respectively, $p=0.3720)$ and hospital survivors and non-survivors (1/48 $(2.1 \%)$ and $1 / 19(5.3 \%)$, respectively, $p=0.4906)$.

Eight children received surgery for residual lesions during ECMO and all were successfully weaned. Two children who had a mitral valve replacement or an arterial switch by the Aubert procedure were unable to be discharged. After weaning, four children had additional surgery and one with recurrent pulmonary venous obstruction after repair of TAPVC was unable to be discharged.

\section{Uni-variate and Multi-variate Logistic Regression Analyses for Survival of ECMO or Hospital Discharge (Tables 8, 9, 10)}

ECMO weaning was affected by heterotaxy, bleeding complications, and several clinical variables during support. Weight, support time, circuit exchange, and bleeding impacted hospital survival. Seven factors during post-ECMO affected hospital discharge despite successful decannulation.
After adjusting for all covariates selected by a stepwise model, high lactate, high total bilirubin, and FNFB during support were independent factors associated with increased mortality of ECMO. Only FNFB after weaning impacted hospital survival independently.

\section{Intermediate Outcomes}

A total of 39 children survived, but 8 died by the end of the follow-up period. 12 out of 39 received surgeries as follows: TCPC in 4 non-HLHS patients after BCPS, BCPS in 3 HLHS patients after the Norwood stage I, neoaortic arch plasty in one non-HLHS patient after the Norwood stage I, and one patient each with RV-pulmonary artery conduit replacement in truncus arteriosus with central A-P shunt and DORV/PA, release of pulmonary venous obstruction in post-TAPVC repair, and atrioventricular valve plasty in a post-CAVSD correction.

Eight children had received no additional surgery during their hospital discharge and death. Three children who had d-TGA associated with CHARGE syndrome, IAA/ DORV/AS, and truncus arteriosus combined with a partial anomalous pulmonary venous connection died of cardiac arrest and 2 died suffering from respiratory distress from an RS viral infection. The cause of death was undetected in 3 children.

The age and body weight of survivors and nonsurvivors after hospital discharge were $20.7 \pm 32.8$ and $5.2 \pm 7.0$ months $(p=0.0359)$ and $9.1 \pm 6.7$ and $4.6 \pm 1.9 \mathrm{~kg}(p=0.0127)$, respectively. The period of hospitalization of survivors and non-survivors was $61.1 \pm 41.1$ and $93.4 \pm 51.9$ days $(p=0.0816)$, respectively. 
Table 8 Uni-variate logistic regression analysis about demographic and during ECMO variables for survival risk factors of ECMO and hospital discharge

\begin{tabular}{|c|c|c|c|c|c|c|}
\hline & \multicolumn{3}{|c|}{ Survival from ECMO } & \multicolumn{3}{|c|}{ Survival to hospital discharge } \\
\hline & Odds ratio & $95 \% \mathrm{CI}$ & $p$ value & Odds ratio & $95 \% \mathrm{CI}$ & $p$ value \\
\hline Body weight (kg) & 1.12 & $0.95-1.45$ & 0.2102 & 1.22 & $1.03-1.53$ & $0.0188^{*}$ \\
\hline Heterotaxy (yes:no) & 0.18 & $0.05-0.65$ & $0.0107 *$ & 0.91 & $0.22-4.62$ & 0.9011 \\
\hline $\mathrm{CRP}(\mathrm{mg} / \mathrm{dl})$ & 0.90 & $0.82-0.97$ & $0.0107 *$ & 0.96 & $0.87-1.06$ & 0.4316 \\
\hline Lactate (mg/dl) & 0.97 & $0.95-0.99$ & $0.0052^{*}$ & 0.99 & $0.97-1.01$ & 0.2248 \\
\hline Creatinine (mg/dl) & 0.16 & $0.03-0.69$ & $0.0139 *$ & 0.56 & $0.12-2.42$ & 0.4213 \\
\hline Total bilirubin (mg/dl) & 0.78 & $0.65-0.89$ & $<0.0001^{*}$ & 0.85 & $0.69-1.01$ & 0.0621 \\
\hline Urinary output (ml/kg/h) & 3.6 & $1.48-11.35$ & $0.0018^{*}$ & 1.23 & $0.87-2.08$ & 0.2256 \\
\hline FNFB (day) & 0.59 & $0.45-0.75$ & $<0.0001^{*}$ & 0.75 & $0.51-1.06$ & 0.1069 \\
\hline ECMO duration (day) & 0.83 & $0.70-0.95$ & $0.0008 *$ & 0.87 & $0.75-0.99$ & $0.0309 *$ \\
\hline Circuit exchange (number) & 0.19 & $0.06-0.45$ & $0.0001 *$ & 0.35 & $0.14-0.82$ & $0.0148 *$ \\
\hline Wall motion (mild-mod.:severe-akine.) & 6.93 & $1.92-29.07$ & $0.0031 *$ & 2.92 & $0.87-9.87$ & 0.0835 \\
\hline Bleeding (yes:no) & 0.24 & $0.06-0.86$ & $0.0281 *$ & 0.25 & $0.09-0.66$ & $0.0054 *$ \\
\hline
\end{tabular}

CRP C-reacting protein, ECMO extracorporeal membrane oxygenation, FNFB the day of first negative fluid balance, mild-mod. mild-moderate hypokinesis, severe-akine. severe hypokinesis-akinesis, $W B C$ white blood cell

${ }^{*} p$ value $<0.05$ was considered significant

Table 9 Uni-variate logistic regression analysis about ECMO duration and postECMO variables for survival risk factors of hospital discharge

\begin{tabular}{llrr}
\hline & \multicolumn{2}{l}{ Survival to hospital discharge } \\
\cline { 2 - 4 } \cline { 3 - 4 } & Odds ratio & $95 \%$ CI & $p$ value \\
\hline ECMO duration (day) & 0.89 & $0.78-1.00$ & 0.0539 \\
WBC $\left(\times 10^{3} \mu / \mathrm{ml}\right)$ & 0.99 & $0.99-0.99$ & $0.0185^{*}$ \\
Lactate $(\mathrm{mg} / \mathrm{dl})$ & 0.96 & $0.93-0.98$ & $0.0038^{*}$ \\
Creatinine $(\mathrm{mg} / \mathrm{dl})$ & 0.15 & $0.03-0.48$ & $0.0004^{*}$ \\
Urinary output $(\mathrm{ml} / \mathrm{kg} / \mathrm{h})$ & 2.64 & $1.53-5.78$ & $<0.0001^{*}$ \\
FNFB (day) & 0.59 & $0.37-0.83$ & $<0.0001^{*}$ \\
Wall motion (mild-mod.:severe-akine.) & 6.13 & $1.09-47.6$ & $0.0400^{*}$ \\
Peritoneal dialysis (yes:no) & 0.03 & $0.001-0.15$ & $<0.0001^{*}$ \\
\hline
\end{tabular}

$E C M O$ extracorporeal membrane oxygenation, FNFB the day of first negative fluid balance, mild-mod. mild-moderate hypokinesis, severe-akine. severe hypokinesis-akinesis, $W B C$ white blood cell

$* p$ value $<0.05$ was considered significant

\section{Cox Proportional Hazard Analyses for Risk Factors of Survival After Hospital Discharge (Table 11)}

Body weight at the time of ECMO-related surgery affected outcomes. In the multivariate model, for each increasing $1 \mathrm{~kg}$ of body weight, the adjusted hazard risk of death after hospital discharge was 0.58 .

\section{Discussion}

ECMO is an integral part of treatment for neonates and children after surgery for congenital heart disease. However, ECMO use is still associated with significant morbidity and mortality. ECMO support of these patients is $2.2-14 \%$ and the survival rate is $33-67 \%[2,5-8,11,16]$, which are similar to our findings of $3.8 \%$ and $61 \%$, respectively. Several factors that influence survival, such as body weight and age, have been identified. The high mortality in neonates weighing less than $3 \mathrm{~kg}$ and needing postcardiotomy ECMO is affected by immature substrate-depleted myocardium and pulmonary vascular hypertension $[2,3,5,17]$. We observed a trend toward poor survival in patients younger than 10.6 months of age and $<5.5 \mathrm{~kg}$ for early outcomes. Furthermore, young age and low weight were observed in non-survivors after hospital discharge. Alsoufi et al. reported that low-weight infants were at an increased mortality risk after cardiac surgery for both early and long-term outcomes [18]. The hazard of death in this subset of children is prolonged and continues for 1-2 years before normalizing. A 
Table 10 Multi-variate logistic regression analysis for survival risk factors of ECMO and hospital discharge

\begin{tabular}{|c|c|c|c|c|c|c|}
\hline & \multicolumn{3}{|c|}{ Survival from ECMO } & \multicolumn{3}{|c|}{ Survival to hospital discharge } \\
\hline & $\begin{array}{l}\text { Adjusted odds } \\
\text { ratio }\end{array}$ & $95 \% \mathrm{CI}$ & $p$ value & $\begin{array}{l}\text { Adjusted odds } \\
\text { ratio }\end{array}$ & $95 \% \mathrm{CI}$ & $p$ value \\
\hline \multicolumn{7}{|l|}{ Pre-ECMO } \\
\hline Body weight (kg) & & & & 1.14 & $0.89-1.57$ & 0.3342 \\
\hline \multicolumn{7}{|l|}{ During ECMO } \\
\hline Lactate $(\mathrm{mg} / \mathrm{dl})$ & 0.97 & $0.93-0.98$ & $0.0019^{*}$ & & & \\
\hline Total bilirubin (mg/dl) & 0.84 & $0.65-0.99$ & $0.0417 *$ & & & \\
\hline FNFB (day) & 0.42 & $0.18-0.67$ & $<0.0001 *$ & & & \\
\hline \multicolumn{7}{|l|}{ Post-ECMO } \\
\hline FNFB (day) & & & & 0.65 & $0.38-0.92$ & $0.0060 *$ \\
\hline Peritoneal dialysis (yes:no) & & & & 0.17 & $0.04-1.68$ & 0.1739 \\
\hline Wall motion (mild-mod.:severe-akine.) & & & & 3.45 & $0.24-56.16$ & 0.3556 \\
\hline
\end{tabular}

$E C M O$ extracorporeal membrane oxygenation, $F N F B$ the day of first negative fluid balance, mild-mod. mild-moderate hypokinesis, severe-akine. severe hypokinesis-akinesis, $W B C$ white blood cell

${ }^{*} p$ value $<0.05$ was considered significant

Table 11 Cox proportional hazard analysis for risk factor of survival after hospital discharge

\begin{tabular}{|c|c|c|c|c|c|c|}
\hline & \multicolumn{3}{|l|}{ Univariable } & \multicolumn{3}{|l|}{ Multivariable } \\
\hline & Hazard ratio & $95 \% \mathrm{CI}$ & $p$ value & $\begin{array}{l}\text { Adjusted hazard } \\
\text { ratio }\end{array}$ & $95 \% \mathrm{CI}$ & $p$ value \\
\hline \multicolumn{7}{|l|}{ Pre-ECMO } \\
\hline Body weight (kg) & 0.69 & $0.43-0.94$ & $0.0119 *$ & 0.58 & $0.25-0.95$ & $0.0215^{*}$ \\
\hline \multicolumn{7}{|l|}{ During ECMO } \\
\hline ECMO duration (day) & 1.12 & $0.97-1.27$ & 0.1025 & 1.21 & $0.88-1.85$ & 0.2265 \\
\hline Circuit exchange (number) & 1.41 & $0.45-3.42$ & 0.5119 & & & \\
\hline Bleeding (yes:no) & 0.73 & $0.04-4.11$ & 0.7607 & & & \\
\hline \multicolumn{7}{|l|}{ Post-ECMO } \\
\hline $\mathrm{WBC}\left(\times 10^{3} \mu / \mathrm{ml}\right)$ & 0.99 & $0.99-1.00$ & 0.2431 & & & \\
\hline Lactate (mg/dl) & 1.03 & $0.99-1.07$ & 0.1514 & 1.11 & $0.99-1.32$ & 0.0581 \\
\hline Creatinine (mg/dl) & 0.60 & $0.03-2.96$ & 0.6157 & & & \\
\hline Urinary output (ml/kg/h) & 0.90 & $0.57-1.24$ & 0.5552 & & & \\
\hline FNFB (day) & 1.42 & $0.89-2.24$ & 0.1366 & 1.22 & $0.66-2.26$ & 0.5152 \\
\hline Peritoneal dialysis (yes:no) & 1.48 & $0.27-7.98$ & 0.6348 & & & \\
\hline Wall motion (mild-mod.:severe-akine.) & 0.30 & $0.05-5.61$ & 0.3314 & & & \\
\hline
\end{tabular}

$E C M O$ extracorporeal membrane oxygenation, $F N F B$ the day of first negative fluid balance, mild-mod. mild-moderate hypokinesis, severe-akine. severe hypokinesis-akinesis, $W B C$ white blood cell

${ }^{*} p$ value $<0.05$ was considered significant

higher incidence of prematurity and associated extracardiac congenital and genetic abnormalities are involved with this outcome. Our adjusted hazard ratio of 0.58 for survival after discharge was similar to that of 0.51 for their risk analysis model.

Survival in this subset of patients is strongly influenced by the type of congenital heart disease and the complexity of the cardiac surgical procedure. Some single-ventricle physiologies are associated with worse prognosis, but others have found that bi-ventricular disease patients have a lower survival rate $[8,19,20]$. Single-ventricle physiology is related to poorer outcomes for ECMO survival, which is $29-48 \%$ $[2,6,21,22]$. This may reflect the particular challenge of balancing systemic and pulmonary circulation on ECMO to prevent pulmonary over circulation, myocardial ischemia, low cardiac output, end-organ injury, and volume loading of the single ventricle. In our study, the survival rate of ECMO weaning and hospital discharge of single ventricle was $81 \%$ 
and $61 \%$, respectively. Our better outcomes depend on the management of systemic-to-pulmonary shunt of Norwood stage I and the modification of cannulation technique to suit cavopulmonary shunt cases. In case of decreasing systemic perfusion despite increasing ECMO flow, the shunt which usually left open during ECMO was adjusted its diameter by a surgical clip to limit pulmonary blood flow. In patients with cavopulmonary shunts, bicaval direct cannulation of outflow cannula was effective for better ECMO operation with sufficient drainage of venous blood. However, attention must be paid to a single-ventricle morphology in which a heterotaxy and a TAPVC coexist. Five (42\%) of the ECMO non-survivors had a single ventricle associated with heterotaxy and 4 had combined TAPVC. For heterotaxy with a single functional ventricle, concomitant TAPVC repair was significantly associated with higher hospital mortality, in addition to postoperative ECMO and need for reoperation [23].

ECMO duration is a strong determinant not only for weaning but also after decannulation [7, 11, 24]. Myocardial function among surviving post-cardiotomy patients returns within 3-5 days and is unlikely to improve after 8-10 days of support [6, 25, 26]. Consistent with other studies, both ECMO and hospital non-survivors needed longer support time. For weaning, more than 9 days of mean support time was a risk for hospital survival in this study. The presence of an oxygenator increases the foreign body surface area of the system, resulting in increased activation of the inflammatory cascade, consumption of coagulation factors, hemolysis, and generation of thromboemboli, which contribute to end-organ damage [5, 27]. When continuation of mechanical support is necessary without an oxygenator, conversion to a VAD should be considered early in selected children as an exit strategy of bridge-to-recover or bridge-to-bridge. With at least 6 days of support, ECMO allows better patient selection for myocardial recovery, VAD implantation, or heart transplantation [28]. On the other hand, children with primarily respiratory failure after cardiac surgery may benefit from longer ECMO. In our study, one patient on a 20-day ECMO run with Norwood palliation with severe pulmonary edema survived to discharge after 4 months of hospitalization and was alive after 33 months of follow-up.

Improved survival in children requiring mechanical support for the inability to separate from CPB has also been reported $[8,29]$. In our study, $81 \%$ of ECMO operations were in this subset and $63 \%$ of supported patients survived to discharge. Before progressive decline in clinical status despite administering abundant inotropic agents, mechanical support should be initiated. It is obvious that ameliorating oxygenation of an impaired lung after CPB improves cardiac function and systemic acidosis. Early ECMO support, such as initiation in the OR, probably decreases the incidence of cardiac arrest and end-organ damage.
Multi-variable risk analysis identified that high levels of lactate, high levels of total bilirubin, and unable to obtain early FNFB during ECMO were independent risk factors associated with ECMO weaning. Late FNFB after successful decannulation increased the risk of hospital death. Lactate is a surrogate marker of tissue perfusion that is influenced by not only macro-circulation but also micro-circulation [30]. ECMO therapy may affect liver perfusion due to changes in pump flow, lack of pulsatility, and rapid changes of vasopressor doses after ECMO initiation [31]. Hemodynamic instability associated with hypoxic hepatitis in ECMO patients may explain the pronounced impact of total bilirubin on clinical outcome.

FNFB is a clinical expression of fluid overload (FO). FO induced by a systemic inflammatory response (SIR) and capillary leak from the deleterious effects of CPB is a sensitive and specific predictor of adverse outcomes in pediatric cardiac surgery. Moreover, SIR may cause alterations in regional macrovascular and microvascular blood flow and vasomotor tone, often leading to a hyperdynamic, vasodilated state while on ECMO [32-34]. Therefore, FO complicates pediatric postcardiotomy ECMO and is associated with organ dysfunction, prolonged support times, and increased mortality. We found that WBC, which consisted mostly of neutrophils, markedly increased after ECMO in both hospital survivors and non-survivors. Furthermore, the post-ECMO WBC of hospital non-survivors was significantly higher. SIR during ECMO results in widespread activation of the endothelium. Activated vascular endothelium secretes pro-inflammatory cytokines, increases reactive oxygen species, and causes an increased infiltration of neutrophils, which may be responsible for the end-organ damage associated with ECMO [35, 36]. Even after successful decannulation, SIR seemed to progress. About $60 \%$ of patients developed SIR syndrome (SIRS) after ECMO decannulation. SIRS continued for about 1 week after ECMO support, and half of patients with SIRS after weaning have a new infection [34]. To modulate SIR during ECMO, various biocompatibility strategies are used to suppress neutrophil-endothelium interactions, such as bio-coating and minimized oxygenator or circuits [37].

Unable to dispense with dialysis is associated with worse prognosis and reflects secondary organ injury due to cardiovascular collapse. Our use of primary PD as a renal replacement therapy during ECMO was consistent with others [32, 38]. Early aggressive fluid removal should benefit children through reduction of organ edema and decreasing ECMO duration by maintaining perfusion of a critical organ.

Bleeding complications that required red blood cell transfusion (RBCT) during an ECMO run from a surgical cause or coagulopathy negatively affected decannulation and hospital discharge, similar to others [11, 24, 39]. RBCT improves circulatory instability and oxygen delivery; 
however, this benefit may be countered by the risk of transfusion-associated lung injury, immune modulation increasing the incidence of infections, cellular hypoxia, and other unknown factors.

MOF and/or sepsis occurred in 58\% of deaths before hospital discharge despite no difference in observed SOFA scores between survivors and non-survivors. Longer ECMO support often needs an exchange of circuits because of clot formation or declining oxygenator function. Circuit exchange requires another round of RBC for priming, which carries an additional risk of SIR. The number of circuit exchanges impacted both ECMO weaning and hospital discharge and should be minimized to limit the volume of newly primed blood and to shorten suspension of the ECMO operation.

\section{Study Limitations}

Our study is retrospective and many variables were not controlled despite good predictions from multi-variable models. The cohort was small, which limited detection of significant differences in outcomes within subgroup analyses. Although many potential factors were evaluated for their impact on outcomes, additional risk variables that may affect outcomes, such as hemodynamic variables, ventilatory parameters, and chemical mediators, were not evaluated. In addition, we did not analyze outcomes after hospital discharge in detail despite a long median follow-up period. Because the investigation occurred at a single center study, it is difficult to generalize these findings without further evaluation of a large number of patients in a multicenter fashion.

\section{Conclusions}

ECMO is beneficial for children after a cardiac operation, but morbidity and mortality remain high. Therefore, it is important to examine risk factors that predict poor outcomes for ECMO weaning, hospital discharge, and after discharge. High levels of lactate, high levels of total bilirubin, and unable to obtain early FNFB were independent risks for ECMO weaning. The primary pathophysiologies of children under postcardiotomy ECMO were hypoperfusion of organs and systemic FO induced by cardiopulmonary distress, and SIR. A longer period of ECMO because of unrecovered cardiac function may necessitate a change in strategy, such as bridge-to-bridge, in conditions of acceptable oxygenation within 9 days of support to prevent a SIR cascade. Late FNFB in the period of post-ECMO increased the risk of death after successful decannulation. FNFB may serve as composite outcome that incorporates the cumulative effects of these morbidities. Surviving to hospital discharge after successful weaning should be strongly affected not only by secondary cardiopulmonary shock associated with ECMO separation but also by protracted SIR. Therefore, careful management of SIR during ECMO runs is needed. Body weight at the time of ECMO-related surgery affected intermediate outcomes. Despite successful ECMO and survival to hospital discharge, children with a lower age and longer hospitalization also need careful outpatient surveillance and management.

\section{Compliance with Ethical Standards}

Conflict of interest There is no conflict of interest to be disclosed.

Ethical Approval All procedures performed in studies involving human participants were in accordance with the ethical standards of the institutional and/or national research committee and with the 1964 Helsinki declaration and its later amendments or comparable ethical standards.

Informal Consent For this type of study formal consent is not required

\section{References}

1. Ravishankar C, Dominguez TE, Kreutzer J, Wernovsky G, Marino BS, Godinez R et al (2006) Extracorporeal membrane oxygenation after stage I reconstruction for hypoplastic left heart syndrome. Pediatr Crit Care Med 7:319-323

2. Bhat P, Hirsch JC, Gelehrter S, Cooley E, Donohue J, King K et al (2013) Outcomes of infants weighing three kilograms or less requiring extracorporeal membrane oxygenation after cardiac surgery. Ann Thorac Surg 95:656-662

3. Haines NM, Rycus PT, Zwischenberger JB, Bartlett RH, Undar A (2009) Extracorporeal life support registry report 2008: neonatal and pediatric cardiac cases. ASAIO J 55:111-116

4. Thiagarajan RR, Barbaro RP, Rycus PT, Mcmullan M, Conrad SA, Fortenberry JD et al (2017) Extracorporeal organization registry international report 2016. ASAIO J 63:60-67

5. Alsoufi B, Shen I, Karamlou T, Giacomuzzi C, Burch G, Silberbach $\mathrm{M}$ et al (2005) Extracorporeal life support in neonates, infants, and children after repair of congenital heart disease: modern era results in a single institution. Ann Thorac Surg 80:15-21

6. Kolovos NS, Bratton SL, Moler FW, Bove EL, Ohye RG, Bartlett RH et al (2003) Outcome of pediatric patients treated with extracorporeal life support after cardiac surgery. Ann Thorac Surg 76:1435-1442

7. Baslaim G, Bashore J, Al-Malki F, Jamjoom A (2006) Can the outcome of pediatric extracorporeal membrane oxygenation after cardiac surgery be predicted? Ann Thorac Cardiovasc Surg 12:21-27

8. Aharon AS, Drinkwater DC Jr, Churchwell KB, Quisling SV, Reddy VS, Taylor M et al (2001) Extracorporeal membrane oxygenation in children after repair of congenital cardiac lesions. Ann Thorac Surg 72:2095-2102

9. Alsoufi B, Al-Radi OO, Gruenwald C, Lean L, Williams WG, McCrindle BW et al (2009) Extra-corporeal life support following cardiac surgery in children: analysis of risk factors and survival in a single institution. Eur J Cardiothorac Surg 35:1004-1011

10. Polimenakos AC, Wojtyla P, Smith PJ, Rizzo V, Nater M, El Zein $\mathrm{CF}$ et al (2011) Post-cardiotomy extracorporeal cardiopulmonary resuscitation in neonates with complex single ventricle: analysis of outcomes. Eur J Cardiothorac Surg 40:1396-1405 
11. Kumar TKS, Zurakowski D, Dalton H, Talwar S, Allard-Picou A, Duebener LF et al (2010) Extracorporeal membrane oxygenation in postcardiotomy patients: factors influencing outcome. J Thorac Cardiovasc Surg 140:330-336

12. Kelly RB, Harrison RE (2010) Outcome predictors of pediatric extracorporeal cardiopulmonary resuscitation. Pediatr Cardiol $31: 626-633$

13. Chang WW, Tsai FC, Tsai TY, Chang CH, Jenq CC, Chang MY et al (2012) Predictors of mortality in patients successfully weaned from extracorporeal membrane oxygenation. PLoS ONE 7:e42687

14. Singer M, Deutschman CS, Seymour CW, Shankar-Hari M, Annane D, Bauer M et al (2016) The third international consensus definitions for sepsis and septic shock (sepsis-3). JAMA 315:801-810

15. Allan CK, Thiagarajan RR, del Nido PJ, Roth SJ, Almodovar MC, Laussen PC (2007) Indication for initiation of mechanical circulatory support impacts survival of infants with shunted singleventricle circulation supported with extracorporeal membrane oxygenation. J Thorac Cardiovasc Surg 133:660-667

16. Sasson L, Cohen I, Tamir A, Sternfeld AR, Berlowitz Y, Lenczner O et al (2013) Extracorporeal membrane oxygenation in pediatric patients: our experience in the last ten years. IMAJ 15:13-16

17. Gelehrter S, Fifer CG, Armstrong A, Hirsch J, Gajarski R (2011) Outcomes of hypoplastic left heart syndrome in low-birth- weight infants. Pediatr Cardiol 32:1175-1181

18. Alsoufi B, Manlhiot C, Mahle WT, Kogon B, Border WL, Cuadrado A et al (2014) Low-weight infants are at increased mortality risk after palliative or corrective cardiac surgery. J Thorac Cardiovasc Surg 148:2508-2514

19. Ford MA, Gauvreau K, McMullan DM, Almodovar MC, Cooper DS, Rycus PT et al (2016) Factors associated with mortality in neonates requiring extracorporeal membrane oxygenation for cardiac indications: analysis of the Extracorporeal Life Support Organization Registry data. Pediatr Crit Care Med 17:860-870

20. Kulik TJ, Moler FW, Palmisano JM, Custer JR, Mosca RS, Bove EL et al (1996) Outcome-associated factors in pediatric patients treated with extracorporeal membrane oxygenator after cardiac surgery. Circulation 94(9 Suppl):II63-II68

21. Salvin JW, Laussen PC, Thiagarajan RR (2008) Extracorporeal membrane oxygenation for postcardiotomy mechanical cardiovascular support in children with congenital heart disease. Pediatr Anesth 18:1157-1162

22. Gajarski RJ, Mosca RS, Ohye RG, Bove EL, Crowley DC, Custer JR et al (2003) Use of extracorporeal life support as a bridge to pediatric cardiac transplantation. J Heart Lung Transplant 22:28-34

23. Alsoufi B, McCracken C, Schlosser B, Sachdeva R, Well A, Kogon B (2016) Outcomes of multistage palliation of infants with functional single ventricle and heterotaxy syndrome. J Thorac Cardiovasc Surg 151:1369-1377

24. Balasubramanian SK, Tiruvoipati R, Amin M, Aabideen KK, Peek GJ, Sosnowski AW et al (2007) Factors influencing the outcome of pediatric cardiac surgical patients during extracorporeal circulatory support. J Cardiothorac Surg 2:4-12
25. Montgomery VL, Strotman JM, Ross MP (2000) Impact of multiple organ system dysfunction and nosicomial infections on survival of children treated with extracorporeal membrane oxygenation after heart surgery. Crit Care Med 28:526-531

26. Kocis K (2000) Pediatric cardiac extracorporeal membrane oxygenation: supporting life or prolonging death? Crit Care Med 28:594-595

27. Darling EM, Kaemmer D, Lawson DS, Jaggers JJ, Ungerleider RM (2001) Use of ECMO without the oxygenator to provide ventricular support after Norwood stage I procedures. Ann Thorac Surg 71:735-736

28. Rousse N, Juthier F, Pinçon C, Hysi I, Banfi C, Robin E et al (2015) ECMO as a bridge to decision: recovery, VAD, or heart transplantation? Int J Cardiol 187:620-627

29. Jaggers JJ, Forbess JM, Shah AS, Meliones JN, Kirshbom PM, Miller CE et al (2000) Extracorporeal membrane oxygenation for infant postcardiotomy support: significance of shunt management. Ann Thorac Surg 69:1476-1483

30. Marik PE, Baram M, Vahid B (2008) Does central venous pressure predict fluid responsiveness? A systematic review of the literature and the tale of seven mares. Chest 134:172-178

31. Bellomo R, Auriemma S, Fabbri A, D'Onofrio A, Katz N, McCullough PA et al (2008) The pathophysiology of cardiac surgery-associated acute kidney injury (CSA-AKI). Int J Artif Organs 31:166-178

32. Wilder NS, Yu S, Donohue JE, Goldberg CS, Blatt NB (2016) Fluid overload is associated with late poor outcomes in neonates following cardiac surgery. Pediatr Crit Care Med 17:420-427

33. Hazle MA, Gajarski RJ, Yu S, Donohue J, Blatt NB (2013) Fluid overload in infants following congenital heart surgery. Pediatr Crit Care Med 14:44-49

34. Thangappan K, Cavarocchi NC, Baram M, Thoma B, Hirose H (2016) Systemic inflammatory response syndrome (SIRS) after extracorporeal membrane oxygenation (ECMO): incidence, risks and survivals. Heart Lung 45:449-453

35. Millar JE, Fanning JP, McDonald CI, McAuley DF, Fraser JF (2016) The inflammatory response to extracorporeal membrane oxygenation (ECMO): a review of the pathophysiology. Crit Care 20:387-396

36. McILwain RB, Timpa JG, Kurundkar AR, Holt DW, Kelly DR, Hartman YE et al (2010) Plasma concentrations of inflammatory cytokines rise rapidly during ECMO-related SIRS due to the release of preformed stores in the intestine. Lab Invest 90:128-139

37. Punjabi PP, Taylor KM (2013) The science and practice of cardiopulmonary bypass: from cross circulation to ECMO and SIRS. Glob Cardiol Sci Pract 3:249-260

38. Sasser WC, Robert SM, Askenazi DJ, O’Meara LC, Borasino S, Alten JA (2014) Peritoneal dialysis: an alternative modality of fluid removal in neonates requiring extracorporeal membrane oxygenation after cardiac surgery. JECT 46:157-161

39. Salvin JW, Scheurer MA, Laussen PC, Wypij D, Polito A, Bacha EA et al (2011) Blood transfusion after pediatric cardiac surgery is associated with prolonged hospital stay. Ann Thorac Surg 91:204-211 